\title{
Three-Dimensional Characterization of Weld Defects in a Steel Material
}

\author{
${ }^{1,2} \mathrm{~A}$. Bakdid, ${ }^{1} \mathrm{~B}$. El Kihel, ${ }^{2} \mathrm{~A}$. Nougaoui and ${ }^{3}$ F. Delaunois \\ ${ }^{1}$ Laboratory of Dynamics and Optics of Materials, Department of Physics, Faculty of Sciences, \\ ${ }^{2}$ Industrial and Engineering Laboratory, School of Applied Sciences, \\ University Mohamed First, 60000 Oujda, Morocco \\ ${ }^{3}$ Department of Metallurgy, Faculty of Engineering, UMONS, \\ 56 Rrues de 1 Epargne, 7000 Mons, Belgium
}

\begin{abstract}
The safety and durability of each construction depends primarily on the accuracy of the weld joint. Therefore, it is very important to join the metals without any defects. This study focuses on finding defects in a weld by determining the depth and size of these defects. Experimental work of welding is made in the form of a simple V-groove which we have inspected and analyzed by phased array ultrasonic testing method. This research allowed us to realize that the technique is much better compared to that based on conventional welding, same as to observe that it maximizes structural safety.
\end{abstract}

$\underline{\text { Key words: Welding, defect, phased array, experimental, technique, welding }}$

\section{INTRODUCTION}

Different types of methods are used to join metal parts and welding is one of them. The phased array technique which is an evolution of conventional ultrasound, allows to reduce inspection times and improve data traceability through mapping realization. Phased array are used more particularly for the control of structures of complex shapes or areas that are hardly accessible both in manufacturing and in maintenance (Hoque et al., 2017).

The technique in question finds applications in particular in the control of welding which is the case of our study, tubes, trees with multiple sections or the cartography of thickness (research of corrosion)

Ultrasonic testing of welds has two steps. The first is to obtain a signal regardless of the defect present in the assembly and to be certain that this signal actually corresponds to the presence of a lack of compactness and not the structure or geometry. The second is to determine the characteristics of the defect from the ultrasonic signal returned by the latter (Walaszek, 2013).

Ultrasonic examination by sensors phased array rests on the same physical principle as conventional ultrasonic testing. It consists in highlighting and exploiting the signals returned by discontinuities in the controlled piece (Kopp, 1999).
The particularity of this examination, compared to conventional ultrasound examination, based on the use of ultrasonic phased array sensors.

Thus, thanks to electronics, the multi-element technology makes it possible in particular to vary the refraction angle and change the focal length of the sensor by applying different delay laws to the array of elements. It thus makes it possible with a single multi-element sensor to have virtually a large number of single-element sensors. The multi-element sensors are defined according to the arrangement of the elements constituting the network for example linear, matrix, annular or circular (Michael, 2012). The most common arrangement is the linear array network for which the elements are arranged in a straight line.

\section{MATERIALS AND METHODS}

The material used in this research is mild steel. It contains about $0.05-0.25 \%$ carbon which making it malleable and ductile. Mild steel has a relatively low tensile strength but it is cheap and easy to form and the surface hardness can be increased by carburizing. The thickness of our sample is $20 \mathrm{~mm}$ and the length is $200 \mathrm{~mm}$.

Preparation of welded samples: Our sample is a mild steel part containing two faces, the face 1 contains holes and

Corresponding Author: A. Bakdid, Laboratory of Dynamics and Optics of Materials, Department of Physics, Faculty of Sciences, University Mohamed First, 60000 Oujda, Morocco 
(a)

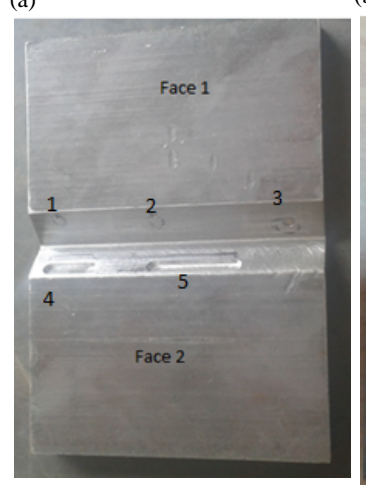

(b)

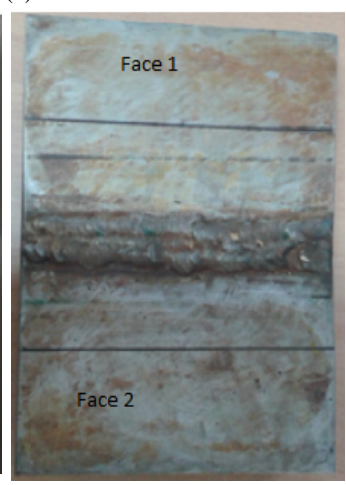

Fig. 1: a, b) Preparation of the weld

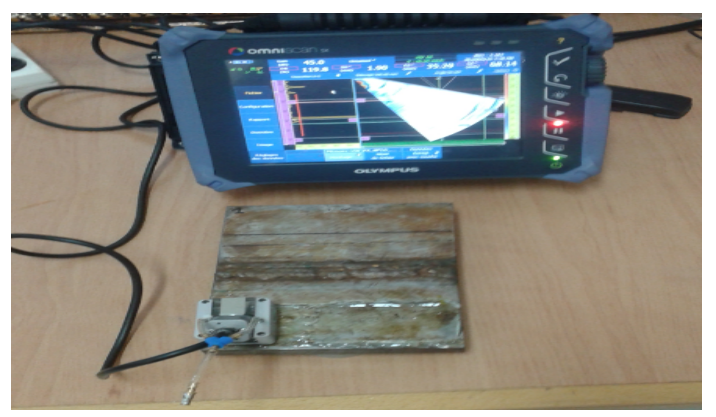

Fig. 2: Experimental set up

the second face contains grooves. This is a simple $\mathrm{V}$ welded by the electric arc (Fig. 1). The sample was prepared and controlled by ultrasonic phased array technology in the laboratory of the P.F.T.2M technology platform of industrial engineering of the National School of Applied Sciences of Oujda.

Experimental set up: In this resaerch, the phased array equipment is composed a Omni-scan SX (Meola et al., 2015) and a 5L32-A11 probe protected by a sabot SA11-N55S, all produced by Olympus Company. This device is perfectly suitable to linear inspections by a straight probe in particular cartography corrosion, composite inspection or again weld inspection a single multi-element group which is the case of our study. The Omni-scan SX is an advanced, multi-technology flaw detector. It is available with PA and conventional UT modules. Contact probes are specially designed to be used directly in contact with the material to be tested. They are longitudinal wave probes and contain composite ceramic which produces high efficiency signals (ASM International, 1989). The scheme below shows some references for sizing the position of a defect in a weld (Bakdid et al., 2015) with (Fig. 2 and 3):

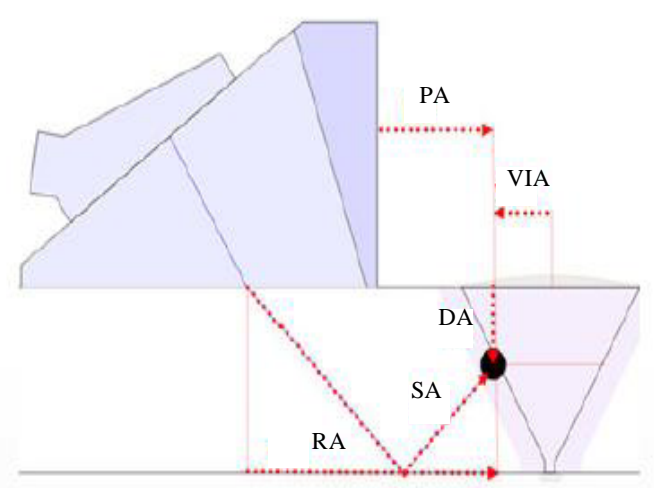

Fig. 3: Dimensions to reference a defect position

- $\mathrm{RA}^{\wedge}$; Distance on the surface of the piece between the point of incidence and the indication detected in door $\mathrm{A}$

- $\mathrm{SA}^{\wedge}$; Sound path from the point of incidence until the indication detected in door $\mathrm{A}$

- $\mathrm{PA}^{\wedge}$; Distance on the surface of the piece between the front face of the wedge (or of the probe) and the indication detected in the door $\mathrm{A}$

- $\mathrm{DA}^{\wedge}$; Depth in the piece of the reflector producing indication detected in door $\mathrm{A}$

VIA ${ }^{\wedge}$; Volumetric position on the index axis of the indication detected in the door $\mathrm{A}$

\section{RESULTS AND DISCUSSION}

In our study, we performed an angular sweep of both faces of the weld in question. The results are presented in the A-scan and S-scan views. The most common format for medical sonograms as well as for phased array images is the S-scan representation which is also called sectorial scan image which is shown as a two-dimensional cross-sectional view. Figure 4-6 of these figures show the scanning face 1 which contains three holes with different sizes $(3,4$ and $6 \mathrm{~mm})$ and depths $(1.5,2$ and $9 \mathrm{~mm})$.

A-scan is a basic representation for an ultrasonic wave and S-scan is another possibility for a data presentation (Bakdid et al., 2017). In Fig. 4, the defect is present at a depth of $\mathrm{DA}^{\wedge}=1.5 \mathrm{~mm}$ and corresponding defect size is $U(m-r)=3.34 \mathrm{~mm}$. In Fig. 5, the defect is present at a depth of $2.41 \mathrm{~mm}$ and its size is $3.66 \mathrm{~mm}$. In Fig. 6, the defect is present at a depth of $8.22 \mathrm{~mm}$ and its size is $6.34 \mathrm{~mm}$.

Regarding the face 2 of the weld, this contains the two grooves with the same size $(7 \mathrm{~mm})$ and the same depth $(1.5 \mathrm{~mm})$. In Fig. 7 , the defect is present at a depth of $1.9 \mathrm{~mm}$ and its size is $7.66 \mathrm{~mm}$. In Fig. 8, the defect is present at a depth of $1.46 \mathrm{~mm}$ and its size is $7.66 \mathrm{~mm}$. 
(a)

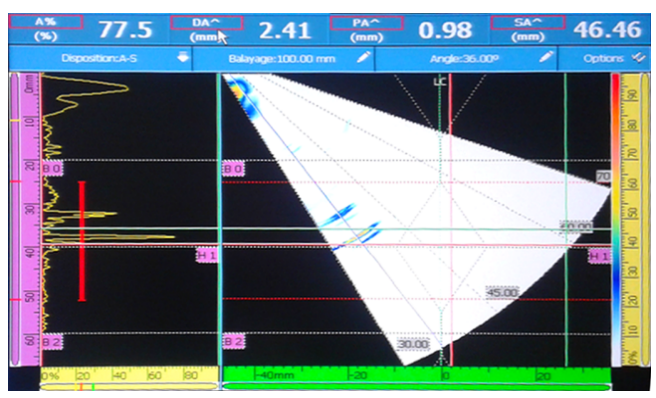

(b)

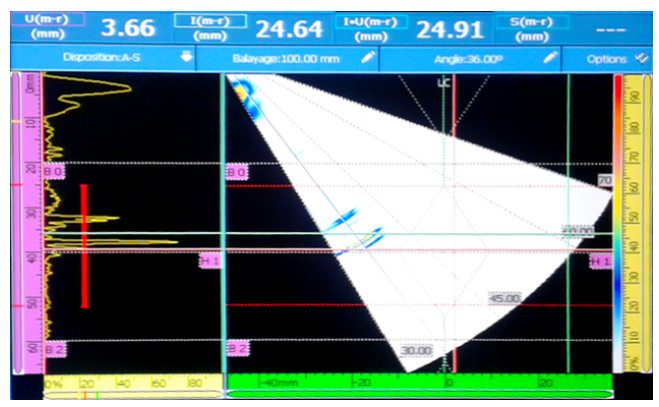

Fig. 4: Sectorial scan of welding a face 1: a) Depth measurement $\mathrm{DA}^{\wedge}$ and b) Size measurement U (m-r) (3.34 mm)
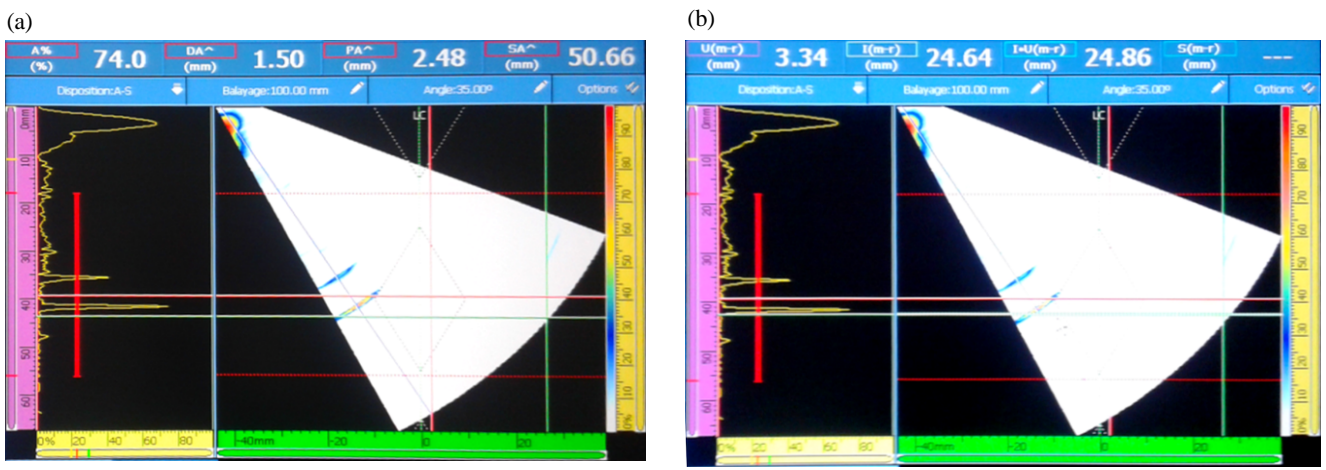

Fig. 5: Sectorial scan of welding a face 1: a) Depth measurement $\mathrm{DA}^{\wedge}$ and b) Size measurement U (m-r) (3.66 mm)

(a)

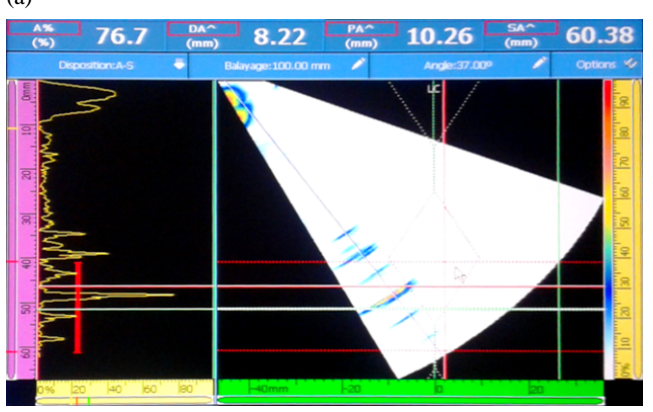

(b)

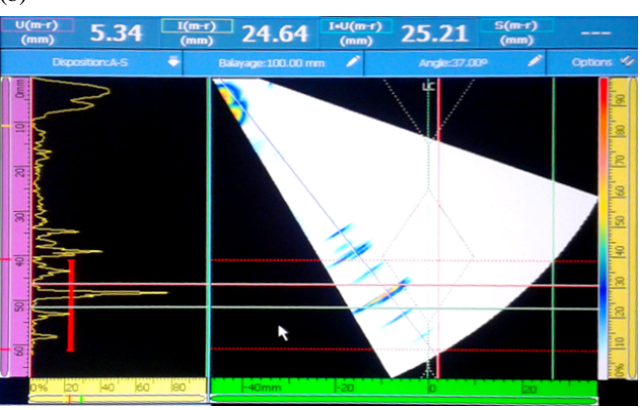

Fig. 6: Sectorial scan of welding a face 1: a) Depth measurement $\mathrm{DA}^{\wedge}$ and b) Size measurement U (m-r) $(8.22 \mathrm{~mm})$
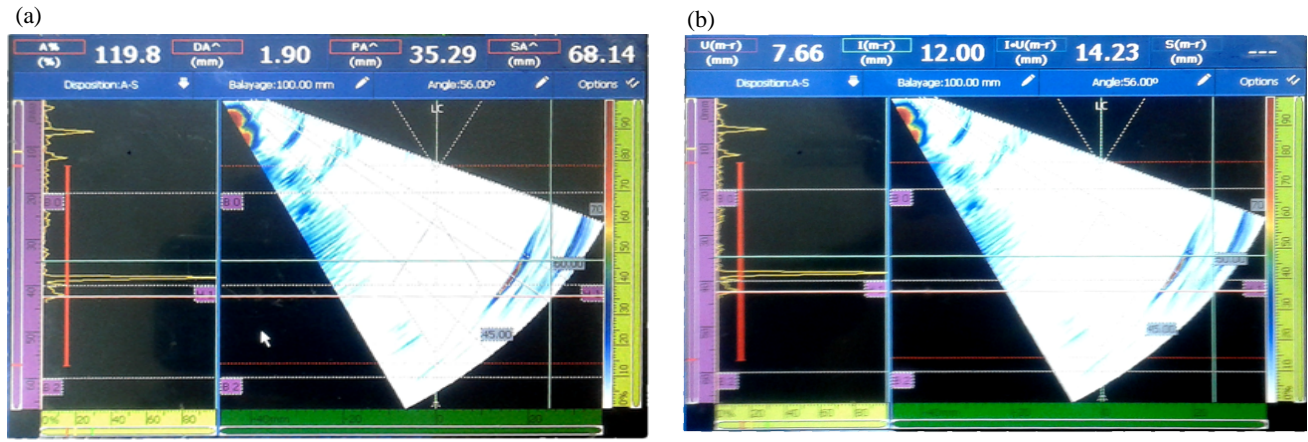

Fig. 7: a, b) Cartography A-scan and S-scan of weld face $2(1.9 \mathrm{~mm})$ 
(a)

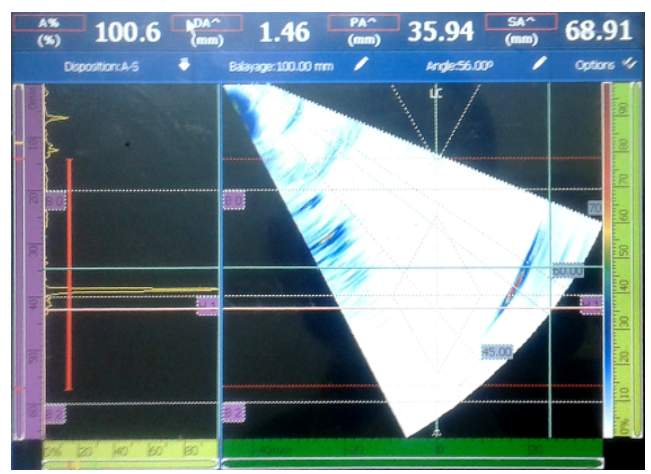

(b)

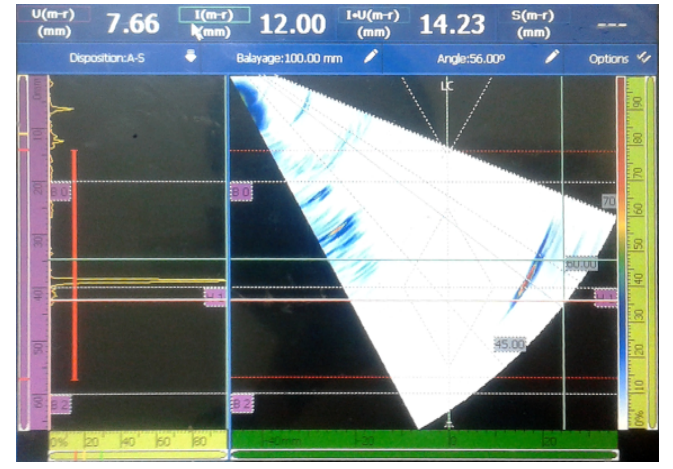

Fig. 8: a, b) Cartography A-scan and S-scan of weld face 2(7.66 mm)

Table 1: Comparison of the experimental results to that of the reality of the measured parameters

\begin{tabular}{lcccccc}
\hline No. of defect & Real depth $(\mathrm{mm})$ & Experimental depth $(\mathrm{mm})$ & Error & Real size $(\mathrm{mm})$ & Experimental size $(\mathrm{mm})$ & Error \\
\hline 1 & 1.5 & 1.50 & 0.00 & 3 & 3.34 \\
2 & 2.0 & 2.41 & 0.41 & 4 & 3.66 \\
3 & 9.0 & 8.22 & 0.78 & 6 & 5.34 \\
4 & 1.5 & 1.90 & 0.40 & 7 & 0.34 \\
5 & 1.5 & 1.46 & 0.04 & 7 & 7.66 \\
\hline
\end{tabular}

The results obtained show that artificial defects in a given location can find an obvious defect image and verify the feasibility of the experimental phased array ultrasonic inspection for welding. The image found by the ultrasound phased array technique contains sufficient information about defects which can explain quite well the location of defects in the weld (Table 1).

From comparison of experimental results to those known before hand as the reality of the pieces, we have come to $t$ he conclusion that the results are very close in a way that their difference is contained in the error which shows the advantage of the technique used for the discovery and the identification of defects.

\section{CONCLUSION}

We used the Omni-scan SX phased array ultrasound technique to determine the size and depth of defects. We have compared this technique with known results beforehand because these defects were created voluntarily by knowing in advance these sizes and depths.

The experimental results we have obtained have shown that the ultrasonic phased array technique for welding has the following advantageous features: scanning flexibility, high detection rate and high efficiency, excellent repeated imaging performance and good. Compared with conventional welding tests, phased array ultrasound technology has exceptional technical advantages.

\section{REFERENCES}

ASM International, 1989. Nondestructive Evaluation and Quality Control. Vol. 17, ASM International, Ohio, Geauga, ISBN:9780871700230, Pages: 795.

Bakdid, A., B. El Kihel, F. Delaunois and A. Nougaoui, 2015. [Technique of control of the welding by the method of ultrasound multi-elements]. Proceedings of the 10th International Conference on Integrated Design and Production, December 2-4, 2015, Tangier, Morocco, pp: 1-8 (In French).

Bakdid, A., D. Bakari, A. Rrhioua, B. EL Kihel and A. Nougaoui et al., 2017. Welding control using ultrasonic multi-elements method. J. Mater. Environ. Sci., 8: 3483-3489.

Hoque, M.E., M.J. Hasan and M.K. Hasan, 2017. Welding joint evaluation of mild steel samples for varying current and voltage to reduce the defect using non destructive testing. Proceedings of the International Conference on Mechanical, Industrial and Materials Engineering (ICMIME2017), December 28-30, 2017, RUET, Rajshahi, Bangladesh, pp: 1-6.

Kopp, N., 1999. [Contribution to the characterization in ultrasonic non-destructive testing of butt welds in carbon steel: Development of a complete automated procedure to the diagnosis]. Ph.D Thesis, Université Paul Verlaine, Metz, France. (In French). 
Meola, C., S. Boccardi, G.M. Carlomagno, N.D. Boffa and E. Monaco et al., 2015. Nondestructive evaluation of carbon fibre reinforced composites with infrared thermography and ultrasonics. Comp. Struct., 134: 845-853.
Michael, M., 2012. Ultrasonic phased array. NDT. Technician, 11: 1-5.

Walaszek, H., 2013. [Non-destructive testing technical book]. Groupe AVRIL, Paris, France. (In French) 